\title{
Multimarket Optimal Bidding for a Power Producer
}

\author{
Miguel A. Plazas, Antonio J. Conejo, Fellow, IEEE, and Francisco J. Prieto
}

\begin{abstract}
This paper considers a profit-maximizing thermal producer that participates in a sequence of spot markets, namely, day-ahead, automatic generation control (AGC), and balancing markets. The producer behaves as a price-taker in both the day-ahead market and the AGC market but as a potential price-maker in the volatile balancing market. The paper provides a stochastic programming methodology to determine the optimal bidding strategies for the day-ahead market. Uncertainty sources include prices for the day-ahead and AGC markets and balancing market linear price variations with the production of the thermal producer. Results from a realistic case study are reported and analyzed. Conclusions are duly drawn.
\end{abstract}

Index Terms-Electricity spot markets, market power, optimal bidding strategies, stochastic programming.

\section{NOTATION}

The notation used throughout the paper is reproduced below for quick reference.

Sets:

$H \quad$ Set of hours.

$J \quad$ Set of generating units.

$K \quad$ Set of scenarios.

$M \quad$ Set of blocks of the revenue function in the balancing market.

Numbers:

$B \quad$ Number of blocks of the revenue function in the balancing market.

$N^{e} \quad$ Number of scenarios for market $e$, where $e=\{\mathrm{D}$ (day-ahead), G (AGC), A (balancing) \}.

$N \quad$ Total number of scenarios.

Constants:

$C_{j} \quad$ Variable operating cost for unit $j$ (Euro/MWh).

$P_{j 0} \quad$ Power output of unit $j$ at the beginning of period 1 (MW).

$P_{j}^{A G C} \quad$ AGC capacity for unit $j$ (MW).

$P_{j}^{\max }$ Capacity of unit $j(\mathrm{MW})$.

$P_{j}^{\min } \quad$ Minimum power output of unit $j(\mathrm{MW})$.

$R_{j}^{d w} \quad$ Ramp-down limit for unit $j(\mathrm{MW} / \mathrm{h})$.

$R_{j}^{u p} \quad$ Ramp-up limit for unit $j(\mathrm{MW} / \mathrm{h})$.

Manuscript received October 15, 2004; revised April 15, 2005. The work of A. J. Conejo was supported in part by the Ministry of Science and Education of Spain under CICYT Project DPI2003-01362 and in part by Junta de Comunidades de Castilla-La Mancha under Project GC-02-006.

M. A. Plazas is with the Unión Fenosa Generación, Madrid, Spain (e-mail: maplazas@uef.es).

A. J. Conejo is with the Department of Electrical Engineering, Universidad Castilla-La Mancha, Ciudad Real, Spain (e-mail: Antonio.Conejo@uclm.es).

F. J. Prieto is with the Department of Statistics, Universidad Carlos III, Madrid, Spain (e-mail: franciscojavier.prieto@uc3m.es).
$T_{h}^{\max } \quad$ Maximum energy to be traded in the balancing market in hour $h(\mathrm{MWh})$.

$\lambda^{\max } \quad$ Upper bound for random variable price (Euro/MWh).

$\pi_{k} \quad$ Probability of scenario $k$.

Random variables:

$\alpha_{h} \quad$ Intercept of the inverse demand curve corresponding to hour $h$ in the balancing market.

$\beta_{h} \quad$ Slope of the inverse demand curve corresponding to hour $h$ in the balancing market.

$\lambda_{h}^{e} \quad$ Price in hour $h$ and market $e$, where $e=\{\mathrm{D}$ (dayahead), G (AGC) $\}$.

It should be noted that a subscript $k$ affecting any of the above variables indicates actual uncertainty realization $k$.

Real variables:

$p_{j h k}^{e} \quad$ Power of unit $j$ in market $e(e=\{D, G, A\})$, hour $h$, and scenario $k(\mathrm{MW})$.

$q_{j h k}^{e} \quad$ Energy produced by unit $j$ in market $e$ and scenario $k$ during hour $h(\mathrm{MWh})$.

$t_{h k}^{e} \quad$ Total energy production (of the generating company) in hour $h$, scenario $k$, and market $e(e=\{D, G, A\})$ (MWh).

$t_{m h k}^{A} \quad$ Block $m$ of the total energy production of the generating company in the balancing market in hour $h$ and scenario $k(\mathrm{MWh})$.

Binary variables:

$w_{j h k} \quad$ It is equal to 1 if unit $j$ is engaged in AGC in hour $h$ and scenario $k$ and 0 otherwise.

Functions:

$g_{h k}^{A}(\cdot) \quad$ Revenues for hour $h$ and scenario $k$ in the balancing market (Euro).

$E_{K}(\cdot)$ Expected value over $K$.

$\Psi_{k}^{e}(\cdot) \quad$ Recourse function associate to stage $e(e=\{D, G, A\})$ and scenario $k$.

$O(\cdot) \quad$ Function that provides the price ranking (increasing value) for all the considered scenarios.

\section{INTRODUCTION}

W E consider a power producer that owns several generating units and participates in a pool-based market framework that includes three independent and successive markets: the day-ahead market, the automatic generation control (AGC) market, and the balancing market. We consider that the producer has no market power capability in the day-ahead and AGC markets, but it can influence price in the volatile balancing market. Not modeling market power in this market is unrealistic and leads to unreasonable results. The objective of the producer is to maximize its expected profits from selling energy in the day-ahead and balancing markets and AGC in the AGC market. 


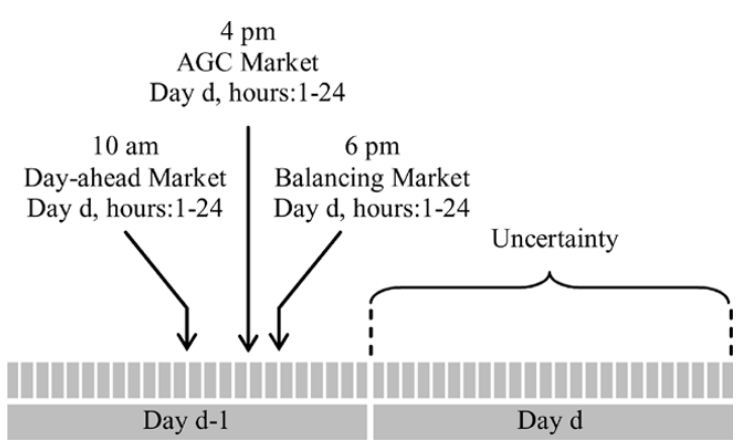

Fig. 1. Time sequence for market clearing.

For instance, in the electricity market of mainland Spain, AGC total revenues are typically $3 \%$ of the energy revenues, but prices range from 16 to 100 Euro/MW (2003) and there exist opportunities for profit spikes. The volume traded in the day-ahead market of mainland Spain in 2004 was Euro 5800 million and the volume negotiated in the AGC market was Euro 128 million. This amount represents only $2.2 \%$ of the energy traded in the day-ahead market but note that AGC is provided at almost no cost to the generating company.

The day-ahead market is cleared through an auction mechanism. That is, producers submit selling bids including energy production blocks and their corresponding minimum selling prices, while consumers submit buying bids consisting in energy consumption blocks and their corresponding maximum buying prices. In turn, the market operator clears the market using a suitable market-clearing procedure [1], [2].

The AGC and balancing markets are cleared using analogous auction procedures. For any of these three markets, market clearing provides hourly prices and accepted production and consumption bids.

The time framework for market clearing is as follows. The day-ahead market concerning the whole day $\mathrm{d}$ is cleared around 10 am of day $d-1$. Analogously, the AGC market concerning the whole day $\mathrm{d}$ is cleared around $4 \mathrm{pm}$ of day $d-1$. The balancing market concerning the whole day $\mathrm{d}$ is cleared around $6 \mathrm{pm}$ of day $d-1$. For these three markets, bids are sent to the market operator just before the closing (clearing) hour, and market-clearing results are known just after that closing hour. This time framework is illustrated in Fig. 1. It should be noted that additional balancing markets can be considered; however, they are generally less relevant than the first balancing market, and this is why they are not considered in this paper. The electricity market of mainland Spain motivates this modeling framework.

This paper provides a methodology that allows a producer to develop appropriate bidding strategies for the day-ahead market, thereby selling its production in the best possible manner. This methodology is based on stochastic programming [3].

It should be noted that the methodology developed can be straightforwardly applied to derive also bidding strategies for the AGC and balancing markets by moving the time window and repeating the procedure. That is, once the day-ahead market results are available, a two-stage stochastic programming problem can be solved to derive bidding stacks for the AGC market.
TABLE I

Stochastic Processes for Prices And Price Functions

\begin{tabular}{cc}
\hline Variable & Weekdays \\
\hline$\lambda_{\mathrm{h}}^{\mathrm{D}}$ & $\operatorname{ARIMA}(1,0,1)(2,0,1)_{24}$ \\
\hline$\lambda_{\mathrm{h}}^{\mathrm{G}}$ & $\operatorname{ARIMA}(5,0,1)(1,0,0)_{24}$ \\
\hline$\alpha_{\mathrm{h}}-\lambda_{\mathrm{h}}^{\mathrm{D}}$ & $\operatorname{ARIMA}(1,0,2)(1,0,1)_{24}$ \\
\hline$\beta_{\mathrm{h}}$ & $\operatorname{ARIMA}(2,0,1)(1,0,1)_{24}$ \\
\hline
\end{tabular}

Also, once the results for the AGC market are also available, a one-stage stochastic programming problem can be solved to derive bidding stacks for the balancing market.

Uncertainty sources include prices for the day-ahead and AGC markets and linear functions describing the way the market price in the balancing market changes with the total production of the producer. We characterize those uncertainties using stochastic models based on historical information.

We consider that self-scheduling is carried out on a weekly basis and therefore the on/off status of the units is known a priori at the time of constructing bidding stacks. Therefore, no start-up and shut-down decisions are considered in this paper.

Relevant references on stochastic programming and electric power are [4]-[8]. Within an electricity market framework, [9] models in detail the day-ahead market but not other markets, and [10] focuses on hydrodominated systems.

Background material on electricity markets can be found in [11]-[15], while background material for stochastic programming is covered in [3], [16], and [17].

This paper is organized as follows. Section II describes the stochastic programming optimization framework that allows deriving optimal bidding strategies for the day-ahead market. In Section III, the stochastic processes involving day-ahead market prices and AGC market prices are characterized. Additionally, the two processes that characterize linearly the price variation with production in the balancing market are characterized. Section IV is a detailed case study where results are reported and analyzed. Some relevant conclusions are drawn in Section V. Section A of the Appendix provides useful definitions, while Section B provides a description of the scenario reduction algorithm used in this paper.

\section{DECISION FRAMEWORK}

This section describes in detail the proposed methodology to derive bidding stacks for the day-ahead market.

\section{A. Scenario Tree}

Prices in the day-ahead market, prices in the AGC market, and price slopes and intercepts in the balancing market are characterized using seasonal ARIMA models. These ARIMA models are provided in Table I. Once those stochastic models have been identified, and their parameters properly fitted using time series data, they can be used to generate price scenarios using a tree format (see Fig. 2). The tree constitutes a discrete and finite approximation of the probability distribution of the stochastic process. The probability associated to a given scenario is the 


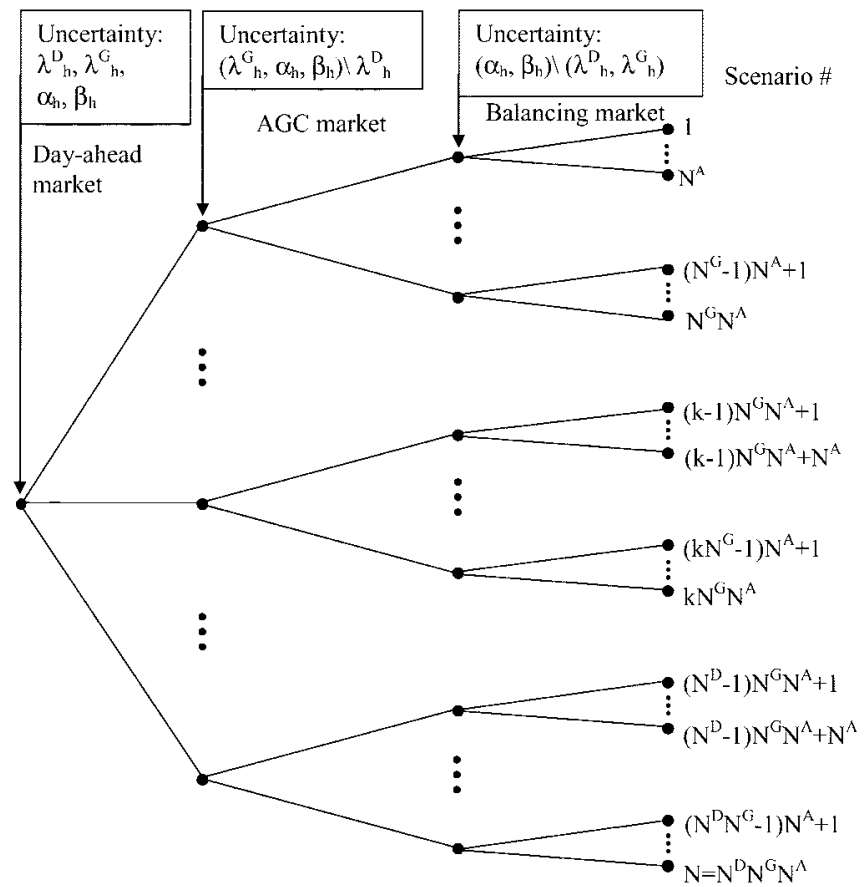

Fig. 2. Scenario tree.

product of the probabilities of occurrence of the prices that constitute the scenario. Note also that those stochastic models capture the dependency of prices across hours.

Therefore, the uncertainty framework is modeled through a symmetric scenario tree [8]. That is, different realizations of prices for the day-ahead market are considered. Then, for each realization of day-ahead market prices, different realizations of the AGC market prices are simulated. Finally, for each realization of the AGC market, different realizations of the two parameters describing the price-production variation of the balancing market are considered.

The number of scenarios for the day ahead market is $N^{D}$ (typically 100) and for the AGC and balancing markets, $N^{G}$ (typically 100) and $N^{A}$ (typically 100), respectively. The total number of scenarios is therefore $N=N^{D} N^{G} N^{A}$ (typically $10^{6}$ ). The scenario tree considered is shown in Fig. 2 .

The scenario tree represented in Fig. 2 can be interpreted as a multistage stochastic process $\xi=\left\{\xi_{e}\right\}_{e=\{D, G, A\}}$, whose probability distribution $P$ is approximated through a finite number of scenarios $\xi^{k}=\left\{\xi_{e}^{k}\right\}_{e=\{D, G, A\}}, k=1, \ldots, N$ with probability $p_{k}, \sum_{k=1}^{N} p_{k}=1$. A scenario reduction technique intended to reduce drastically the number of scenarios while maintaining the statistic properties of the considered stochastic process is carried out [18]. Through this reduction, a subset of scenarios $\left\{k^{[1]}, \ldots, k^{[\ell]}, \ldots, k^{[L]}, L \leq N\right\}$ denoted by $K^{[L]}$ is selected, such that their corresponding probability distribution $P^{[L]}$ is the closest to the original distribution in terms of a certain probability distance between $P$ and $P^{[L]}$.

The technique used to reduce the number of scenarios determines a scenario subset of prescribed cardinality and assigns new probabilities to the preserved scenarios, such that their corresponding probability distribution is the closest to the original distribution in terms of the following probability distance

$$
D\left(P, P^{[L]}\right)=\sum_{\tilde{k} \in K \backslash K^{[L]}} p_{\tilde{k}} \min _{k \in K^{[L]}} \sum_{e}\left|\xi_{e}^{k}-\xi_{e}^{\tilde{k}}\right|
$$

where $e=\{D, G, A\}$ and $|\cdot|$ represents a norm in $\Re^{n}$. Note that the method proposed in this paper uses the scenario-based approximation, which means discrete probability distributions, and considers scenario probabilities as elements of a linear space. Section B of the Appendix provides a heuristic algorithm that approaches the optimal selection of the subset $K^{[L]}$ and assigns new probabilities to the preserved scenarios. Further details can be found in [18]-[20].

For the problem considered in this paper, the scenario reduction technique is first applied to each stage, resulting in $N_{\text {red }}^{D}$, $N_{\text {red }}^{G}$, and $N_{\text {red }}^{A}$ scenarios, respectively, and then to the resulting tree comprising $N_{\text {red }}^{D} N_{\text {red }}^{G} N_{\text {red }}^{A}$ scenarios. This second reduction results in a small number (around 25) of scenarios. For computing expression (1), the quadratic norm 2 is used.

\section{B. Optimization Framework}

The mathematical programming models to be solved to determine the optimal bidding strategies for the power producer in the different spot markets are stated below.

The day-ahead bidding strategy is determined through the following stochastic programming model:

$$
\begin{aligned}
& \text { Maximize } p_{j h k}^{D}, q_{j h k}^{D}, t_{h k}^{D} \\
& E_{K}\left\{\sum_{h}\left(t_{h k}^{D} \lambda_{h k}^{D}-\sum_{j} C_{j} q_{j h k}^{D}\right)\right. \\
& \left.\quad+E_{K \backslash \lambda_{h k}^{D}}\left\{\Psi_{k}^{G}\left(p_{j h k}^{D}, q_{j h k}^{D}, t_{h k}^{D}\right)\right\}\right\}
\end{aligned}
$$

subject to

$$
\begin{aligned}
q_{j h k}^{D}= & \frac{1}{2}\left(p_{j(h-1) k}^{D}+p_{j h k}^{D}\right) \forall j, \forall h, \forall k \\
t_{h k}^{D}= & \sum_{j} q_{j h k}^{D} \forall h, \forall k \\
q_{j h k}^{D}-q_{j h \tilde{k}}^{D} \leq & 0 \forall k, \tilde{k}:\left(O\left(\lambda_{h k}^{D}\right)+1=O\left(\lambda_{h \tilde{k}}^{D}\right)\right), \forall h, \\
& \forall j \\
p_{j h k}^{D}= & p_{j h \tilde{k}}^{D} \forall k, \tilde{k}:\left(\lambda_{h k}^{D}=\lambda_{h \tilde{k}}^{D} \forall h\right), \forall j
\end{aligned}
$$

The objective function (2) includes revenues obtained from the day-ahead market plus expected revenues from AGC and balancing markets. These expected revenues are computed through the problem (7)-(12) below. The expectation operator is taken over AGC and balancing random variables.

Equation (3) defines energies as average values of powers for every scenario. Equation (4) determines total hourly productions for each scenario. Equation (5) is explained below. Equation (6) is an nonanticipativity constraints.

The purpose of the technique proposed in this paper is not to compute the optimal quantities (in a stochastic programming sense) to be sold in the day-ahead market (e.g., $40 \mathrm{MWh}$ in hour 
1, $25 \mathrm{MWh}$ in hour 2, etc.) but rather to derive optimal bidding curves for every hour of the day-ahead market (see Figs. 6 and 7 in Section IV). Therefore, to obtain such curves, variables $\left(p_{j h k}^{D}, q_{j h k}^{D}, t_{h k}^{D}\right)$ are made dependent on scenarios [9] and (5) are introduced to ensure higher productions at higher prices. These conditions make bidding curves nondecreasing, which is a bidding requirement in most markets. In order to obtain the optimal quantity to be sold in the day-ahead market in a standard stochastic programming framework, (6) should be replaced by the nonanticipativity constraint $p_{j h k}^{D}=p_{j h \tilde{k}}^{D} \forall k, \tilde{k} \in K$. It should be noted that this last constraint is more restrictive than constraint (6) because it enforces nonanticipativity for all markets, while (6) only enforces nonanticipativity for the AGC and balancing markets.

The AGC market strategy is determined through the problem below.

$$
\begin{aligned}
& \text { Maximize } \\
& \qquad \begin{array}{l}
p_{j h k}^{G}, q_{j h k}^{G}, t_{h k}^{G}, w_{j h k} \\
\sum_{h} t_{h k}^{G} \lambda_{h k}^{G}+E_{K \backslash\left(\lambda_{h k}^{D}, \lambda_{h k}^{G}\right)} \\
\quad\left\{\Psi_{k}^{A}\left(p_{j h k}^{D}, q_{j h k}^{D}, t_{h k}^{D}, p_{j h k}^{G}, q_{j h k}^{G}, t_{h k}^{G}, w_{j h k}\right)\right\}
\end{array}
\end{aligned}
$$

subject to

$$
\begin{aligned}
q_{j h k}^{G}= & \frac{1}{2}\left(p_{j(h-1) k}^{G}+p_{j h k}^{G}\right) \forall j, \forall h, \forall k \\
q_{j h k}^{G} \leq & w_{j h k} P_{j}^{A G C} \forall j, \forall h, \forall k \\
t_{h k}^{G}= & \sum_{j} q_{j h k}^{G} \forall h, \forall k \\
p_{j h k}^{G}= & p_{j h \tilde{k}}^{G} \forall k, \tilde{k}:\left(\left(\lambda_{h k}^{D}, \lambda_{h k}^{G}\right)=\left(\lambda_{h \tilde{k}}^{D}, \lambda_{h \tilde{k}}^{G}\right) \forall h\right), \\
& \forall j \\
w_{j h k} \in & \{0,1\} \forall j, \forall h, \forall k .
\end{aligned}
$$

The objective function (7) includes revenues obtained from the AGC market plus expected revenues from the balancing market. These expected revenues are computed through the problem (13)-(22) below. The expectation operator is taken over the random variables of the balancing market.

Equation (8) expresses energy values as the average of the powers at the beginning and the end of any considered hour and scenario. Equation (9) limits the amount of power devoted to AGC during any hour and scenario by any generating unit. Equation (10) expresses the total energy associated to AGC activity for every hour and scenario. Equation (11) is an nonanticipativity constraint. Equation (12) declares binary variables that define which units are in AGC operation.

Note that using a binary variable per unit is necessary to differentiate whether the unit is contributing to AGC or ramping. These binary variables complicate the solution of the problem, but they are needed to reflect the actual functioning of generating units. It should be noted that a machine providing AGC cannot ramp up or down as its control is transferred to the ISO. This is common practice in the electricity market of mainland Spain, as stated in the corresponding operating policy enforced by the ISO and reported in [21]. Moreover, the gaps between the power output of a machine providing AGC and its upper and lower bounds, respectively, must allow both up and down control.

Similarly to the day-ahead market, it should be noted that variables $\left(p_{j h k}^{G}, q_{j h k}^{G}, t_{h k}^{G}\right)$ are made dependent on scenarios, which is not the case in a standard stochastic programming framework. This is consistent with using a two-stage model (AGC and balancing markets) similar to the one presented in this paper to generate AGC bidding curves. Equation (11) enforces nonanticipativity only with respect to the balancing market. In a conventional stochastic programming approach, nonanticipativity constraints would enforce nonanticipativity with respect to both the AGC and balancing markets.

The balancing market strategy is determined through the problem below.

$$
\begin{gathered}
\text { Maximize } p_{j h k}^{A}, q_{j h k}^{A}, t_{h k}^{A} \\
\sum_{h}\left(g_{h k}^{A}-\sum_{j} C_{j} q_{j h k}^{A}\right)
\end{gathered}
$$

subject to

$$
\begin{aligned}
& q_{j h k}^{D}+q_{j h k}^{A}+\frac{1}{2} q_{j h k}^{G} \leq P_{j}^{\max } \forall j, \forall h, \forall k \\
& q_{j h k}^{D}+q_{j h k}^{A}-\frac{1}{2} q_{j h k}^{G} \geq P_{j}^{\min } \forall j, \forall h, \forall k \\
& p_{j h k}^{D}+p_{j h k}^{A} \leq P_{j}^{\max } \forall j, \forall h, \forall k \\
& p_{j h k}^{D}+p_{j h k}^{A} \geq P_{j}^{\min } \forall j, \forall h, \forall k \\
& p_{j h k}^{D}+p_{j h k}^{A}-\left(p_{j(h-1) k}^{D}+p_{j(h-1) k}^{A}\right) \\
& \leq\left(1-w_{j h k}\right) R_{j}^{u p} \forall j, \forall h, \forall k \\
& p_{j(h-1) k}^{D}+p_{j(h-1) k}^{A}-\left(p_{j h k}^{D}+p_{j h k}^{A}\right) \\
& \leq\left(1-w_{j h k}\right) R_{j}^{d w} \forall j, \forall h, \forall k \\
& q_{j h k}^{A}=\frac{1}{2}\left(p_{j(h-1) k}^{A}+p_{j h k}^{A}\right) \forall j, \forall h, \forall k \\
& t_{h k}^{A}=\sum_{j} q_{j h k}^{A} \forall h, \forall k \\
& g_{h k}^{A}=t_{h k}^{A}\left(\alpha_{h k}+\beta_{h k} t_{h k}^{A}\right) \forall h, \forall k .
\end{aligned}
$$

The objective function (13) is the revenue obtained from the balancing market.

Equation (14)-(17) sets the maximum (capacity) and minimum power output of any generating unit at any hour and scenario, including AGC activity (if any). Equation (18)-(19) enforces, respectively, up and down ramping limits for generating units not engaged in AGC for every scenario. Equation (20) expresses energy values as an average of the powers at the beginning and the end of any considered hour for every scenario. Equation (21) expresses the total energy associated to the balancing market in every hour and scenario. Equation (22) expresses the balancing market revenue for each hour and scenario as a quadratic concave function. This function is linearized below.

It should be noted that variables $\left(p_{j h k}^{A}, q_{j h k}^{A}, t_{h k}^{A}\right)$ are made dependent on scenarios to achieve bidding curves, which is not the case in a standard stochastic programming framework. No nonanticipativity constraint is required in this last stage. 
Note also that the variables $q$ can be eliminated from the models above using (3), (8), and (20), thus reducing the number of variables required in the formulation.

\section{Equivalent Formulation as a Mathematical Program With Recourse}

The above stochastic programming problem is equivalent to the deterministic one stated below (mathematical program with recourse). This version is readily solvable using an appropriate mixed-integer linear programming solver.

$$
\begin{aligned}
& \sum_{k}^{\operatorname{maximize}}\left[\sum_{h}\left(t_{h k}^{D} \lambda_{h k}^{D}-\sum_{j} C_{j} q_{j h k}^{D}\right)\right] \pi_{k} \\
& +\sum_{k}\left(\sum_{h} t_{h k}^{G} \lambda_{h k}^{G}\right) \pi_{k} \\
& +\sum_{k}\left[\sum_{h}\left(g_{h k}^{A}-\sum_{j} C_{j} q_{j h k}^{A}\right)\right] \pi_{k} .
\end{aligned}
$$

Conditions that ensure a monotonously increasing bidding stack for the day-ahead market are

$q_{j h k}^{D}-q_{j h \tilde{k}}^{D} \leq 0 \forall k, \tilde{k}:\left(O\left(\lambda_{h k}^{D}\right)+1=O\left(\lambda_{h \tilde{k}}^{D}\right)\right) \forall h, \forall j$.

Nonanticipativity constraints for the day-ahead market are

$$
p_{j h k}^{D}=p_{j h \tilde{k}}^{D} \forall k, \tilde{k}:\left(\lambda_{h k}^{D}=\lambda_{h \tilde{k}}^{D} \forall h\right) \forall j .
$$

Nonanticipativity constraints for the AGC market are

$$
\begin{aligned}
p_{j h k}^{G}= & p_{j h \tilde{k}}^{G} \\
& \forall k, \tilde{k}:\left(\left(\lambda_{h k}^{D}, \lambda_{h k}^{G}\right)=\left(\lambda_{h \tilde{k}}^{D}, \lambda_{h \tilde{k}}^{G}\right) \forall h\right) \forall j .
\end{aligned}
$$

Energy balance in the spot (day-ahead, AGC, and balancing) markets are

$$
\begin{aligned}
t_{h k}^{e} & =\sum_{j} q_{j h k}^{e} \forall h, \forall k, e \in\{D, G, A\} \\
q_{j h k}^{e} & =\frac{1}{2}\left(p_{j(h-1) k}^{e}+p_{j h k}^{e}\right) \forall j, \forall h, \forall k, \\
e & \in\{D, G, A\} .
\end{aligned}
$$

Generating unit functioning constraints are

$$
\begin{aligned}
& q_{j h k}^{D}+q_{j h k}^{A}+\frac{1}{2} q_{j h k}^{G} \leq P_{j}^{\max } \forall j, \forall h, \forall k \\
& q_{j h k}^{D}+q_{j h k}^{A}-\frac{1}{2} q_{j h k}^{G} \geq P_{j}^{\min } \forall j, \forall h, \forall k \\
& p_{j h k}^{D}+p_{j h k}^{A} \leq P_{j}^{\max } \forall j, \forall h, \forall k \\
& p_{j h k}^{D}+p_{j h k}^{A} \geq P_{j}^{\min } \forall j, \forall h, \forall k \\
& q_{j h k}^{G} \leq w_{j h k} P_{j}^{A G C} \forall j, \forall h, \forall k \\
& p_{j h k}^{D}+p_{j h k}^{A}-\left(p_{j(h-1) k}^{D}+p_{j(h-1) k}^{A}\right) \\
& \leq\left(1-w_{j h k}\right) R_{j}^{u p} \forall j, \forall h, \forall k \\
& p_{j(h-1) k}^{D}+p_{j(h-1) k}^{A}-\left(p_{j h k}^{D}+p_{j h k}^{A}\right) \\
& \leq\left(1-w_{j h k}\right) R_{j}^{d w} \forall j, \forall h, \forall k \\
& w_{j h k} \in\{0,1\} \forall j, \forall h, \forall k .
\end{aligned}
$$

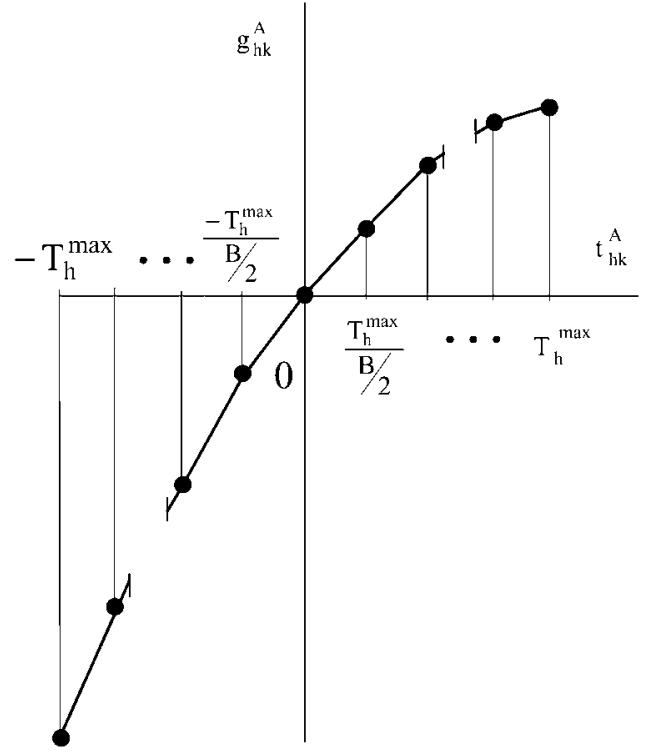

Fig. 3. Illustration of the linearization provided by (37)-(40).

The piece-wise linear (concave) representation of revenues in the balancing market is achieved through the following constraints:

$$
\begin{aligned}
g_{h k}^{A} & =\sum_{m=1}^{B}\left(\alpha_{h k}+\frac{2 T_{h}^{\max }(2 m-(B+1)) \beta_{h k}}{B}\right) t_{m h k}^{A} \\
& \forall h, \forall k \\
\frac{-T_{h}^{\max }}{\frac{B}{2}} & \leq t_{m h k}^{A} \leq 0, \forall m \leq \frac{B}{2}, \forall h, \forall k \\
0 & \leq t_{m h k}^{A} \leq \frac{T_{h}^{\max }}{\frac{B}{2}}, \forall m>\frac{B}{2}, \forall h, \forall k \\
t_{h k}^{A} & =\sum_{m=1}^{B} t_{m h k}^{A}, \forall t_{h k}^{A} \in\left(-T_{h}^{\max }, T_{h}^{\max }\right), \forall h, \forall k .
\end{aligned}
$$

It should be noted that (37)-(40) constitute a straightforward linearization of (22). Fig. 3 illustrates the piece-wise concave linearization carried out through (37)-(40).

It should also be noted that we have experienced better numerical performance using a linear model than a quadratic one.

\section{Solution Procedure}

The problem formulated in the previous section is mixed-integer and linear and can be solved using an appropriate mixedinteger linear programming solver, such as CPLEX [22]. This procedure is used in the case study below.

\section{Characterization of the Stochastic Processes}

Market-clearing prices for the day-ahead and the AGC markets, respectively, are characterized through stochastic processes. Only weekdays are considered. The treatment for weekends is similar and is not reported below.

Analogously, the intercept and slope that describe the participation of the power producer in the balancing market are characterized using stochastic processes. To enhance numerical perfor- 
TABLE II

Characteristics OF THE Thermal Units

\begin{tabular}{cccccccc}
\hline Type & $\begin{array}{c}\mathrm{C}_{\mathrm{j}}^{\mathrm{f}} \\
(\text { Euro/h) }\end{array}$ & $\begin{array}{c}\mathrm{C}_{\mathrm{j}} \\
(\text { Euro } / \\
\mathrm{MWh})\end{array}$ & $\begin{array}{c}\mathrm{P}_{\mathrm{j}}^{\max } \\
(\mathrm{MW})\end{array}$ & $\begin{array}{c}\mathrm{P}_{\mathrm{j}}^{\min } \\
(\mathrm{MW})\end{array}$ & $\begin{array}{c}\mathrm{P}_{\mathrm{j}}^{\mathrm{AGC}} \\
(\mathrm{MW})\end{array}$ & $\begin{array}{c}\mathrm{R}_{\mathrm{j}}^{\text {up }} \\
(\mathrm{MW} / \mathrm{h})\end{array}$ & $\begin{array}{c}\mathrm{R}_{\mathrm{j}}^{\mathrm{dw}} \\
(\mathrm{MW} / \mathrm{h})\end{array}$ \\
\hline Coal_1 & 126.0 & 19.81 & 140 & 75 & 20 & 65 & 65 \\
\hline CCGT_1 & 1097.2 & 25.17 & 380 & 160 & 50 & 220 & 220 \\
\hline CCGT_2 & 992.8 & 25.51 & 390 & 180 & 50 & 210 & 210 \\
\hline Coal_2 & 575.0 & 29.37 & 500 & 250 & 40 & 250 & 250 \\
\hline Oil_1 & 91.5 & 37.91 & 50 & 25 & 0 & 25 & 25 \\
\hline Oil_2 & 1800.0 & 33.91 & 300 & 200 & 20 & 100 & 100 \\
\hline
\end{tabular}

mance, instead of characterizing the hourly intercept, the difference between this intercept and the day-ahead market clearing price is characterized.

It should be noted that the above stochastic processes should generate an adequate number of scenarios, which properly covers the spectrum of possible prices.

All stochastic processes are modeled using seasonal ARIMA models [23]-[25]. The fitted ARIMA models for the week considered in the case study are provided in Table I. Model fitting is ensured using the autocorrelation and partial autocorrelation functions of the error.

Historical data comprising 30 working days are used to fit the models in Table I.

\section{CASE STUdy}

Results from a case study based on the electricity market of mainland Spain [26] are reported in this section. The considered market framework includes the day-ahead market, the AGC market, and the first balancing market [26]. The week under study is June 7-11, 2004 (just working days) in the electricity market of mainland Spain.

It should be noted that in the electricity market of mainland Spain, no virtual bidding is allowed. Settled transactions constitute firm obligations.

The considered producer includes six thermal units whose characteristics are provided in Table II.

Table II includes fixed costs $\left(C_{j}^{F}\right)$. In order to compute daily profit, the constant $24 \times \sum_{j} C_{j}^{F}$ should be subtracted from the revenue of the producer.

The numbers of scenarios for the day-ahead, AGC, and balancing markets are, respectively, 100, 100, and 100. The total number of scenarios is therefore $10^{6}$. The scenario reduction technique is first applied to each stage, resulting in 25,10 , and 10 scenarios, respectively, and then to the resulting tree comprising 2500 scenarios. This second reduction results in just 25 scenarios. Fig. 4 provides a measure of the relative distance between the tree comprising 2500 scenarios and trees containing a reduced number of scenarios for the case of June 7, 2004. The relative distance is defined as the distance measured between the reduced tree and the original tree, divided by the distance measured between a reduced tree that considers just one scenario and the original tree. For this case study, a tree with 25 scenarios provides a reasonable approximation as can be deduced from Fig. 5, which shows how the optimal objective function value changes as the number of scenarios increases.

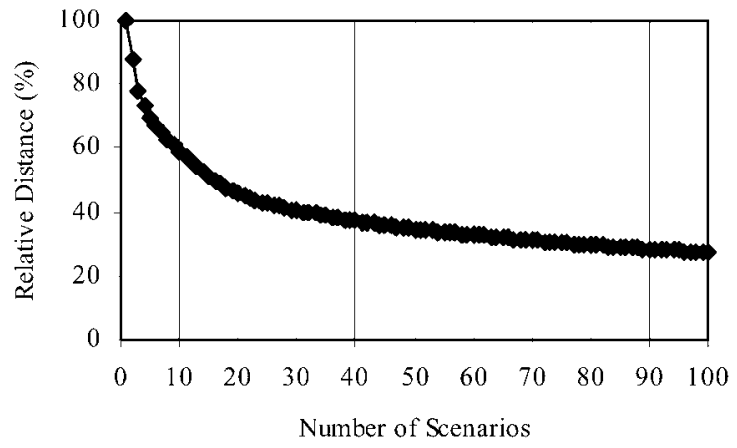

Fig. 4. Relative distance of the original tree to reduced scenario trees.

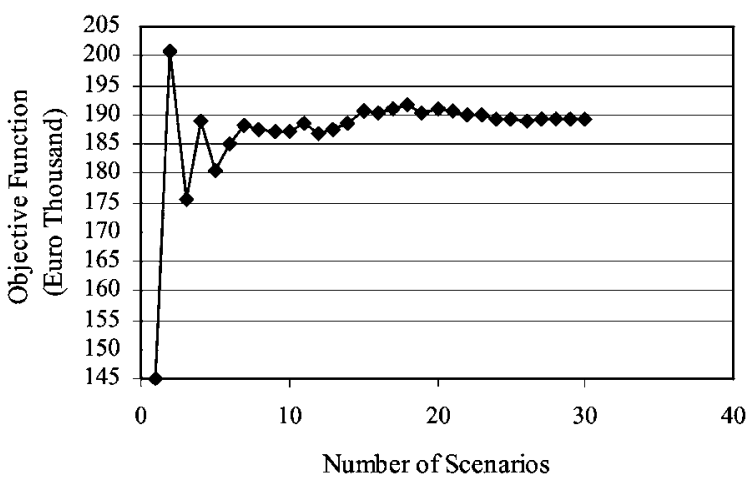

Fig. 5. Objective function value as a function of the number of scenarios considered.

TABLE III SOLUTION TIME AND QUALITY

\begin{tabular}{lc}
\hline \multicolumn{2}{c}{ Monday, June 7, 2004 } \\
\hline CPU time (s) & 100 \\
\hline Absolute gap (Euro) & 834 \\
\hline Relative gap (\%) & 0.44 \\
\hline
\end{tabular}

We have also analyzed in detail the best use of Benders decomposition to address the problem of interest, but our implementations have only produced frustrating results due to the size of the master problem. We decided not to try Lagrangian techniques because of their lack of robustness associated with their required tune-up of parameters.

The number of constraints, real variables, and binary variables in the resulting problem are, respectively, 44833, 22 201, and 3600. It has been solved using CPLEX under GAMS [22]. The computational characteristics of the solution attained are illustrated in Table III. The CPU time needed to attain the solution using a Dell PowerEdge 6600 with two processors at $1.60 \mathrm{GHz}$ and $2 \mathrm{~Gb}$ of RAM memory is $100 \mathrm{~s}$.

The optimal profit values attained (for the three markets) using alternative approaches are reported in Table IV.

To obtain a measure of quality for the results attained if the technique proposed would have been applied to the actual electricity market situation of mainland Spain on June 7, 2004, and to compare these results with those obtained using other methods, the solutions below are considered.

Stochastic solution: Once day-ahead market bidding curves are obtained using the proposed model, energies for the dayahead market are fixed considering actual price values (June 7, 
TABLE IV

PROFIT (EURO THOUSAND)

\begin{tabular}{cc}
\hline \multicolumn{2}{c}{ Monday, June 7, 2004 } \\
\hline Stochastic & 487.0 \\
\hline Deterministic & 481.8 \\
\hline Classic & 482.2 \\
\hline
\end{tabular}

2004) for that market. Then, the proposed model is run considering the fixed energy values for the day-ahead market and only one scenario for the AGC and balancing markets comprising the actual price values (June 7, 2004). The result obtained constitutes a measure of the maximum benefit that the company can achieve in all markets if bidding is carried out according with the proposed model.

Deterministic solution: Using the expected values of all stochastic variables, the resulting deterministic model is run and optimal values for energy in the day-ahead market are determined. Then, energy for the day-ahead market is fixed considering the solution provided by the deterministic model and the proposed (stochastic) model is executed considering only one scenario comprising the actual price values (June 7, 2004) for the AGC and balancing markets. The result obtained constitutes a measure of the maximum benefit that the company can achieve in all markets if bidding is carried out using a deterministic model.

Classic solution: Using the expected values of the stochastic variables, a one-stage deterministic model comprising the dayahead market is run and optimal values for energy in the dayahead market are determined. Then, energy for the day-ahead market is fixed considering the solution provided by the classic model and the proposed (stochastic) model is executed considering only one scenario comprising the actual price values (June $7,2004)$ for the AGC and balancing markets. The result obtained constitutes a measure of the maximum benefit that the company can achieve in all markets if bidding is carried out using a one-stage deterministic model.

Note that the difference between the deterministic and classic models is that the first model considers three stages to provide a solution for the day-ahead market while the second model only considers the day-ahead (one-stage) market.

Observe the profit advantage obtained by using a stochastic approach. The above comparison provides an appropriate measure of how the stochastic model used reflects the real world.

Several stochastic programming indicators are reported in Table V (as formulated in [3]). Once an optimal deterministic solution is obtained for the deterministic problem resulting from substituting all random variables for their corresponding expected values (EV solution), the expected result of using the EV solution (EEV indicator) is computed as the average value over scenarios of the objective function values corresponding to the optimal deterministic solution of the day-ahead market and all scenarios. Indicator RP is computed as the average value over scenarios of the objective function values corresponding to the optimal stochastic solution of the day-ahead market and all scenarios. Indicator VSS is computed as RP minus EEV. Note that VSS is a measure of the advantage obtained if a stochastic model is used rather than a naïve deterministic one. This indicator shows that the solution obtained is of a high quality from
TABLE V

Stochastic PRogramming IndicATORS (EURo THOUSAND)

\begin{tabular}{lc}
\hline \multicolumn{2}{l}{ Monday, June 7, 2004 } \\
\hline RP & 189.6 \\
\hline EEV & 185.6 \\
\hline VSS & 4.0 \\
\hline
\end{tabular}

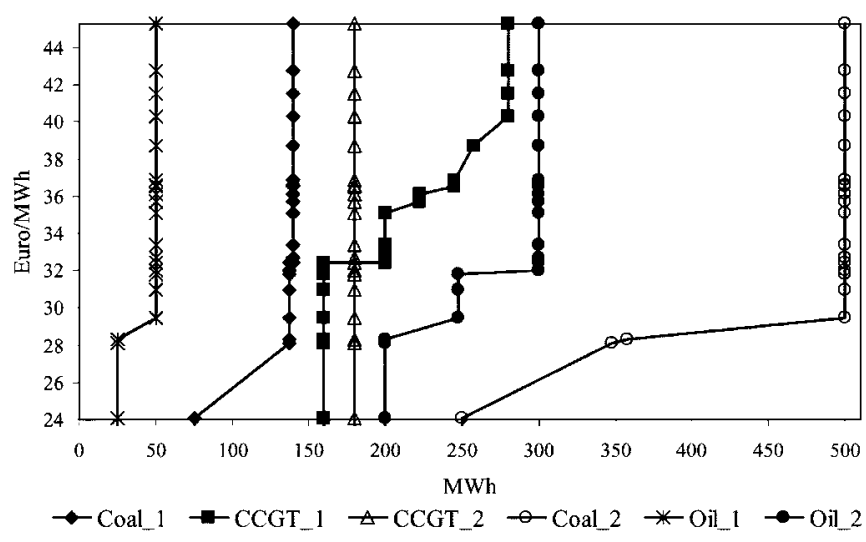

Fig. 6. Bidding stack at period 10 (9 A.M. to 10 A M.) for the day-ahead market.

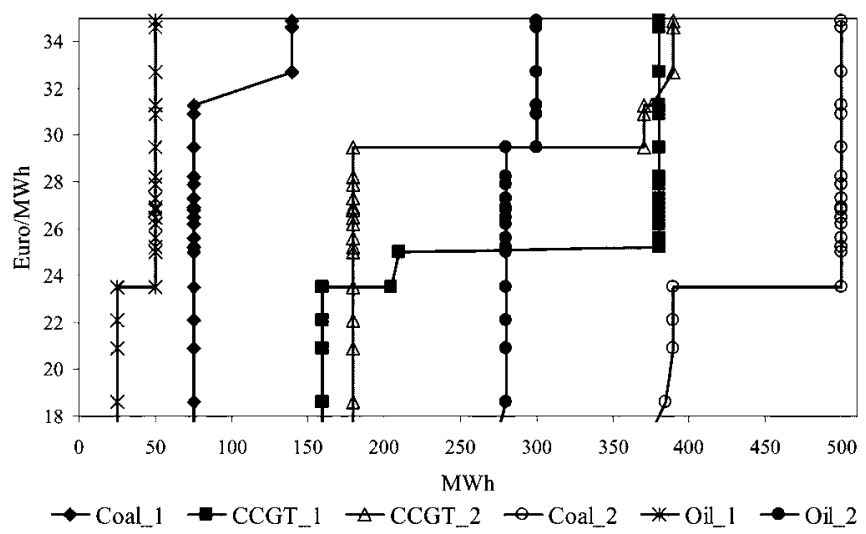

Fig. 7. Bidding stack at period 5 (4 A.M. to 5 A M.) for the day-ahead market.

a stochastic programming viewpoint, and its value shows that it would be possible to attain additional savings of Euro 4000 by taking into account a stochastic solution to the problem.

Fig. 5 shows how the objective function optimal value [(23)] changes as the number or scenarios increases. Note that this value coincides with indicator RP. Observe that a robust solution is attained for 25 or more scenarios and that the scenario reduction illustrated in Fig. 4 is appropriate. It should be noted that a critical issue when using a stochastic programming approach based on scenarios is the actual number of scenarios to consider. A low number of scenarios might result in inaccuracies while a large number implies high computational burden. An appropriate manner to resolve this tradeoff is to increase the number of scenarios until the objective function value stabilizes. This is the criterion used in this case study.

Figs. 6 and 7 provide the optimal bidding stacks for the dayahead market at period 10 (from 9 am to $10 \mathrm{am}$ ) and 5 (4 am to $5 \mathrm{am}$ ), respectively. Bidding stacks for other hours are similar. It should be noted that for a market requiring step-wise bidding stacks (the case of the market in mainland Spain) the linear 
TABLE VI

CHARACTERISTICS OF THE SOLUTION. JUNE 7-11, 2004 (FIgurEs IN EURo THOUSAND)

\begin{tabular}{ccccccc}
\hline June 7-11,2004 & 7 & 8 & 9 & 10 & 11 & Total \\
\hline Stochastic & 487.0 & 367.2 & 96.9 & 53.2 & 293.0 & 1297.2 \\
\hline Deterministic & 481.8 & 358.0 & 97.9 & 56.7 & 291.0 & 1285.4 \\
\hline Classic & 482.2 & 359.5 & 97.9 & 55.4 & 295.1 & 1290.2 \\
\hline RP & 189.5 & 414.0 & 89.6 & 5.8 & 13.9 & 712.9 \\
\hline EEV & 185.6 & 413.2 & 85.6 & 0.5 & 9.6 & 694.5 \\
\hline VSS & 4.0 & 0.8 & 4.0 & 5.3 & 4.3 & 18.4 \\
\hline
\end{tabular}

blocks of the stacks in Figs. 6 and 7 should be approximated by step-wise functions.

For instance, the solution obtained for period 5 (see Fig. 7) results in $85 \mathrm{MWh}$ bought in the balancing market (average value for the solution obtained over the considered 25 scenarios). This value becomes $356 \mathrm{MWh}$ if the same model is run without considering market power in the balancing market. Using the actual price quantity function for period 5, the actual payment is Euro 7798, while not considering market power would result in a payment of Euro 6657. Note the significant differences in energy traded and payments.

Only bidding stacks for the day-ahead market are reported in the paper, as the paper concentrates on the decisions pertaining to that market. Note that in multistage sequential markets, decisions in subsequent markets are made once the actual values of the previous-stage stochastic variables are known.

The solution obtained using the deterministic model for period 5 results in the three cheapest units plus one oil unit working close to their respective upper limits (Coal_1, CCGT_1, CCGT_2, and Oil_1). This solution implies buying back energy in the balancing market since the expected price for this market is comparatively low. However, the actual prices for period 5 in both the day-ahead and balancing markets are lower than the variable cost of most of the units, but buying back in the balancing market the whole surplus of energy is unprofitable due to the influence that this energy would have on the resulting price in the balancing market. On the other hand, considering the bidding curves provided by the stochastic model, the energy sold for the actual day-ahead price in period 5 of 20.4 Euro/MWh is smaller than the energy sold using the deterministic model. Consequently, one concludes that the stochastic solution is a better solution in this particular case.

The whole procedure is repeated for June 7-June 11, 2004 (Monday to Friday). Results (for the three markets) are reported in Table VI, where the advantage of using a multistage stochastic programming approach as the one presented in this paper should be readily apparent. For the working days of the week under study, $0.91 \%$ profit advantage (Euro 11.8 thousand) is obtained with respect to the deterministic approach and $0.54 \%$ (Euro 7.0 thousand) with respect to the classic one. Indicators EEV, RP, and VSS clearly show the advantage of using a multistage stochastic programming approach. Indicator VSS is Euro 18.4 thousand, a significant amount of money.

It can be concluded from Table VI that a stochastic approach clearly outperforms the deterministic and classic ones. Only on June 9 and 10, when forecast price values are close to actual

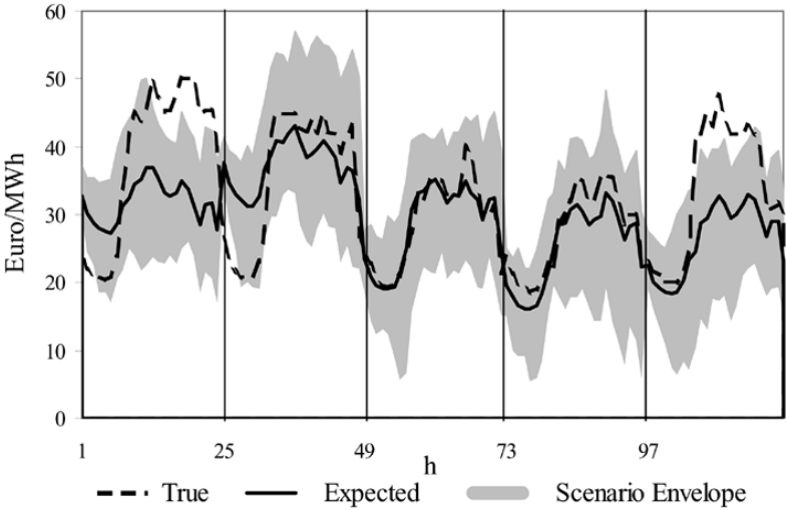

Fig. 8. True and forecast prices and price scenario envelope. June 7-11, 2004.

prices (periods 49 to 96 in Fig. 8), is a deterministic approach more advantageous.

\section{CONCLUSIONS}

This paper provides a procedure to derive optimal bidding stacks for a power producer that participates in the spot electricity markets, including day-ahead, AGC, and balancing. The objective of the producer is to maximize the expected profit from its involvement in the spot markets. The proposed procedure targets a producer with no market power in the day-ahead and AGC markets but with the capability of influencing market-clearing prices in the volatile balancing market. The proposed technique is built within the versatile decision framework provided by the stochastic programming methodology. A robust yet efficient solution technique is used. A real-world case study based on the electricity market of mainland Spain is used to illustrate the appropriate functioning of the procedure developed.

\section{APPENDIX}

This Appendix provides definitions for acronyms and some specific stochastic programming expressions and a mathematical description of the scenario reduction algorithm used [18].

\section{A. Acronyms and Other Definitions}

1) ARIMA: Stochastic model to characterize price behavior.

2) RP: Indicator RP is computed as the average value over scenarios of the objective function values corresponding to the optimal stochastic solution of the day-ahead market and all scenarios.

3) EEV: Indicator EEV is computed as the average value over scenarios of the objective function values corresponding to the optimal deterministic solution of the day-ahead market and all scenarios.

4) VSS: Indicator VSS is computed as RP minus EEV.

5) Nonanticipativity: Nonanticipativity conditions enforce that decisions depend only on temporal information up to the time at which decisions are made.

6) Relative distance: The distance measured between the reduced tree and the original tree, divided by the distance measured between a reduced tree that considers just one scenario and the original tree. 


\section{B. Scenario Reduction Algorithm}

The optimal choice of an index set $K^{[L]}$ for scenario reduction problem with fixed cardinality $\# K^{[L]}$ is given by the solution of the problem

$$
\text { Minimize } \quad\left\{D\left(P, P^{[L]}\right): \# K^{[L]}=L\right\} .
$$

Since this problem is difficult to solve, a heuristic algorithm that approaches the optimal selection of the subset $K^{[L]}$ and assigns new probabilities to the preserved scenarios has been developed in [19]. This algorithm is reproduced below for the reader's convenience. Further details can be found in [18]-[20]. Note that the expression $D\left(P, P^{[L]}\right)$ denotes the value $\min _{k \in K \backslash K[\ell-1]} D_{k}^{[L]}$ computed by the algorithm at step $L$.

The algorithm proceeds as follows.

\section{Step 1)}

Compute

$$
d_{\tilde{k} k}^{[1]}=\sum_{e}\left|\xi_{e}^{k}-\xi_{e}^{\tilde{k}}\right| \forall \tilde{k}, \quad k \in\{1, \ldots, N\}
$$

and

$$
D_{k}^{[1]}=\sum_{\tilde{k} \neq k} \pi_{\tilde{k}} d_{\tilde{k} k}^{[1]} \forall k \in\{1, \ldots, N\} .
$$

Set

and

$$
k^{[1]}: k^{[1]} \in \arg \min _{k \in\{1, \ldots, N\}} D_{k}^{[1]}
$$

$$
K^{[1]}=\left\{k^{[1]}\right\}
$$

Step $\ell$ )

Compute

$$
d_{\tilde{k} k}^{[\ell]}=\min \left\{d_{\tilde{k} k}^{[\ell-1]}, d_{\tilde{k} k k^{[\ell-1]}}^{[\ell-1]}\right\} \forall \tilde{k}, k \in K \backslash K^{[\ell-1]}
$$

and

$$
D_{k}^{[\ell]}=\sum_{\tilde{k} \in K \backslash\left\{K^{[\ell-1]} \cup k\right\}} \pi_{\tilde{k}} d_{\tilde{k} k}^{[\ell]} \forall k \in\{1, \ldots, N\} .
$$

Set

and

$$
k^{[\ell]}: k^{[\ell]} \in \arg \min _{k \in K \backslash K K^{[\ell-1]}} D_{k}^{[\ell]}
$$

$$
K^{[\ell]}=\left\{K^{[\ell-1]} \cup k^{[\ell]}\right\}
$$

Step $L$ )

Compute

$$
d_{\tilde{k} k}^{[L]}=\min \left\{d_{\tilde{k} k}^{[L-1]}, d_{\tilde{k} k k^{[L-1]}}^{[L-1]}\right\} \forall \tilde{k}, \quad k \in K \backslash K^{[L-1]}
$$

and

$$
D_{k}^{[L]}=\sum_{\tilde{k} \in K \backslash\left\{K^{[L-1]} \cup k\right\}} \pi_{\tilde{k}} d_{\tilde{k} k}^{[L]} \forall k \in\{1, \ldots, N\} .
$$

Set

$$
k^{[L]}: k^{[L]} \in \arg \min _{k \in K \backslash K K^{[L-1]}} D_{k}^{[L]}
$$

and

$$
K^{[L]}=K^{[L-1]} \cup k^{[L]} .
$$

The subset $\left\{k^{[1]}, \ldots, k^{[\ell]}, \ldots, k^{[L]}\right\}$ constitutes

an approximation to the optimal selection of the set $K^{[L]}$ for a scenario reduction problem with fixed cardinality \# $K^{[L]}$. Step $L+1$ )

For every scenario $k \in K \backslash K^{[L]}$, compute closestto $(k)=k^{[\ell]}: k^{[\ell]} \in \arg \min _{k[\ell] \in k^{[L]}} \sum_{e}\left|\xi_{e^{k^{[\ell]}}}-\xi_{e}^{k}\right|$.

Set the resulting probability of the scenarios included in $K^{[L]}$ as

$$
\pi_{k^{[\ell]}}^{[L]}=\pi_{k^{[\ell]}}+\sum_{k \in K \backslash K^{[L]}: \operatorname{closestto}(k)=k^{[\ell]}} \pi_{k} .
$$

\section{ACKNOWLEDGMENT}

The authors would like to thank Prof. W. Römisch and A. Eichhorn from Humboldt University in Berlin for valuable comments on scenario reduction.

\section{REFERENCES}

[1] J. M. Arroyo and A. J. Conejo, "Optimal response of a power generator to energy, AGC, and reserve pool-based markets," IEEE Trans. Power Syst., vol. 17, no. 2, pp. 404-410, May 2002.

[2] A. L. Motto, F. D. Galiana, A. J. Conejo, and J. M. Arroyo, "Networkconstrained multiperiod auction for a pool-based electricity market," IEEE Trans. Power Syst., vol. 17, no. 3, pp. 646-653, Aug. 2002.

[3] J. R. Birge and F. Louveaux, Introduction to Stochastic Programming. New York: Springer-Verlag, 1997.

[4] L. F. Escudero, J. L. de la Fuente, C. García, and F. J. Prieto, "Hydropower generation management under uncertainty via scenario analysis and parallel computation," IEEE Trans. Power Syst., vol. 11, no. 2 , pp. 683-689, May 1996.

[5] M. P. Nowak and W. Römisch, "Lagrangian relaxation applied to power scheduling in a hydro-thermal system under uncertainty," Ann. Oper. Res., vol. 100, pp. 251-272, 2000.

[6] S. Takriti, B. Krasenbrink, and L. S.-Y. Wu, "Incorporating fuel constraints and electricity spot prices into the stochastic unit commitment problem," Oper. Res., vol. 48, pp. 268-280, 2000.

[7] S. E. Fleten, S. W. Wallace, and W. T. Ziemba, "Hedging electricity portfolios via stochastic programming," in Decision-Making Under Uncertainty: Energy and Power, A. Ryszczynski and C. Greengard, Eds. New York: Springer-Verlag, 2002.

[8] N. Growe-Kuska, K. C. Kiwiel, M. P. Nowak, W. Römisch, and I. Wegner, "Power management in a hydro-thermal system under uncertainty by Lagrangian relaxation," in Decision-Making under Uncertainty: Energy and Power, A. Ryszczynski and C. Greengard, Eds. New York: Springer-Verlag, 2002.

[9] A. Baillo, M. Ventosa, M. Rivier, and A. Ramos, "Optimal offering strategies for generation companies operating in electricity spot markets," IEEE Trans. Power Syst., vol. 19, no. 2, pp. 745-753, May 2004.

[10] M. V. Pereira, S. Granville, M. Fampa, R. Dix, and L. A. Barroso, "Strategic bidding under uncertainty: A binary expansion approach," IEEE Trans. Power Syst., vol. 20, no. 1, pp. 180-188, Feb. 2005.

[11] M. Ilic, F. Galiana, and L. Fink, Power Systems Restructuring: Engineering and Economics. Boston, MA: Kluwer, 1998.

[12] G. B. Sheblé, Computational Auction Mechanisms for Restructured Power Industry Operation. Boston, MA: Kluwer, 1999. 
[13] M. Shahidehpour and M. Alomoush, Restructured Electric Power Systems. New York: Marcel Dekker, 2001.

[14] M. Shahidehpour, H. Yamin, and Z. Li, Market Operations in Electric Power Systems: Forecasting, Scheduling and Risk Management. New York: Wiley, 2002.

[15] M. Shahidehpour and Y. Wang, Communication and Control in Electric Power Systems. New York: Wiley, 2003.

[16] J. L. Higle and S. Sen, Stochastic Decomposition-A Statistical Method for Large Scale Stochastic Linear Programming. Dordrecht, The Netherlands: Kluwer, 1996.

[17] P. Kall and S. W. Wallace, Stochastic Programming. New York: Wiley, 1995.

[18] N. Gröwe-Kuska, H. Heitsch, and W. Römisch, "Scenario reduction and scenario tree construction for power management problem," in Proc. IEEE Bologna Power Tech., Bologna, 2003.

[19] H. Heitsch and W. Römisch, "Scenario reduction algorithms in stochastic programming," Comput. Optimiz. Appl., vol. 24, pp. 187-206, 2003.

[20] J. Dupacova, N. Gröwe-Kuska, and W. Römisch, "Scenario reduction in stochastic programming: An approach using probability metrics," Math. Prog., ser. A, vol. 95, pp. 493-511, 2003.

[21] (2004) System Operator of the Electricity Market of Mainland Spain. REE. [Online]. Available: http://www.ree.es/.

[22] A. Brooke, D. Kendrick, A. Meeraus, and R. Raman, GAMS: A User's Guide, DC: GAMS Development Corporation, 1998.

[23] G. E. P. Box and G. M. Jenkins, Time Series Analysis Forecasting and Control, 2nd ed. San Francisco, CA: Holden-Day, 1976.

[24] J. Contreras, R. Espínola, F. J. Nogales, and A. J. Conejo, "ARIMA models to predict next-day electricity prices," IEEE Trans. Power Syst., vol. 18 , no. 3 , pp. 1014-1020, Aug. 2003.

[25] F. J. Nogales, J. Contreras, A. J. Conejo, and R. Espínola, "Forecasting next-day electricity prices by time series models," IEEE Trans. Power Syst., vol. 17, no. 2, pp. 342-348, May 2002.
[26] (2004) Market Operator of the Electricity Market of Mainland Spain. OMEL. [Online]. Available: http://www.omel.es/.

Miguel A. Plazas received the Industrial Engineering degree from the Universidad Politécnica de Valencia, Valencia, Spain, in 1995.

$\mathrm{He}$ is currently working in electrical markets as a trader for Unión Fenosa Generación. His main research interest is optimization under uncertainty applied to electricity markets.

Antonio J. Conejo (F'04) received the M.S. degree from the Massachusetts Institute of Technology, Cambridge, in 1987, and the Ph.D. degree from the Royal Institute of Technology, Stockholm, Sweden, in 1990.

$\mathrm{He}$ is currently a Professor of electrical engineering at the Universidad de Castilla-La Mancha, Ciudad Real, Spain. His research interests include control, operations, planning, and economics of electric energy systems, as well as statistics and optimization theory and its applications.

Francisco J. Prieto received the Ph.D. degree from Stanford University, Stanford, CA, in 1989.

$\mathrm{He}$ is currently a Professor in the Statistics Department, Universidad Carlos III de Madrid, Madrid, Spain. His research interests include large-scale optimization, nonlinear programming, and multivariate analysis. 